Article

\title{
Ulam Stability of a Second Linear Differential Operator with Nonconstant Coefficients
}

\author{
Liviu Cădariu ${ }^{1, *,+}\left(\mathbb{D}\right.$, Dorian Popa ${ }^{2,+}\left(\mathbb{D}\right.$ and Ioan Raşa ${ }^{2,+}+(\mathbb{C}$ \\ 1 Department of Mathematics, Politehnica University of Timişoara, Piaţa Victoriei no.2, \\ 300006 Timişoara, Romania \\ 2 Department of Mathematics, Faculty of Automation and Computer Science, Technical University of \\ Cluj-Napoca, Str. Memorandumului, No. 28, 400114 Cluj-Napoca, Romania; \\ dorian.popa@math.utcluj.ro (D.P.); ioan.rasa@math.utcluj.ro (I.R.) \\ * Correspondence: liviu.cadariu-brailoiu@upt.ro \\ + These authors contributed equally to this work.
}

Received: 29 July 2020; Accepted: 25 August 2020; Published: 3 September 2020

check for updates

\begin{abstract}
In this paper, we obtain a result on Ulam stability for a second order differential operator acting on a Banach space. The result is connected to the existence of a global solution for a Riccati differential equation and some appropriate conditions on the coefficients of the operator.
\end{abstract}

Keywords: Ulam stability; linear differential operator; Riccati equation; Banach spaces

MSC: 34A30, 34D10, 39B82

\section{Introduction}

Ulam stability is one of the main topics in functional equation theory. The starting point of Ulam stability was the question formulated by S.M. Ulam during a talk given at Madison University, Wisconsin, and concerns the approximate homomorphisms of a metric group (see [1]). The topic was intensively developed and studied in the last 50 years. For some results, methods, extensions and generalizations of the notion we refer the reader to [1,2].

The first results on Ulam stability for differential equations were obtained by M. Obloza [3] and C. Alsina and R. Ger [4]. T. Miura, S. Miyajima and S. Takahasi proved that the linear differential operator of $n$-th order with constant coefficients is stable in Ulam sense if and only if its characteristic equation has no roots on the imaginary axis [5]. This result was generalized by D. Popa and I. Raşa who obtained a sharp estimate of Ulam constant [6]. Recently, A.R. Baias and D. Popa obtained the best Ulam constant for a second order linear differential operator with constant coefficients [7]. Recall also the result obtained by M. Onitsuka et al. on the best constant of linear differential equation of the first order [8]. For more details on Ulam stability of differential equations and differential operators, we refer the reader to [9-12].

To this moment there are few results on Ulam stability for the linear differential operator of higher order with nonconstant coefficients. This is indeed the reason for which in this paper we deal with Ulam stability of a second order linear differential operator with nonconstant coefficients.

In what follows, let $X$ be a Banach space over $\mathbb{R}$. Let $I \subseteq \mathbb{R}$ be an interval, $a \in I, f \in C^{1}(I, \mathbb{R}), g \in$ $C(I, \mathbb{R})$. By $C^{n}(I, X)$, we denote as usual the set of all $n$-times continuously differentiable functions defined on $I$ with values in the Banach space $X$.

Definition 1. [13] Let $A$ be a linear space over the field $\mathbf{R}$. A function $\rho_{A}: A \rightarrow[0,+\infty]$ is called a gauge on A if: 
1. $\rho_{A}(x)=0$ if and only if $x=0$;

2. $\rho_{A}(\lambda x)=|\lambda| \rho(x)$ for all $x \in A, \lambda \in \mathbf{R}, \lambda \neq 0$.

Let us consider the linear differential operator with nonconstant coefficients $D: C^{2}(I, X) \rightarrow C(I, X)$ given by

$$
D(y)=y^{\prime \prime}+f y^{\prime}+g y, \quad y \in C^{2}(I, X) .
$$

Let $\varphi \in C^{n}(I, X), n \in \mathbb{N}$ and denote

$$
\|\varphi\|_{\infty}=\sup \{\|\varphi(x)\|: x \in I\} .
$$

Then $\|\varphi\|_{\infty}$ is a gauge. We suppose also that $C^{n}(I, X), n \in \mathbb{N}$, are endowed with the same gauge $\|\cdot\|_{\infty}$ for $n=0,1,2$.

Definition 2. The operator $D$ is called Ulam stable if there exists a nonnegative constant $L$ such that for every $\varepsilon>0$ and every $y \in C^{2}(I, X)$ satisfying

$$
\|D(y)\|_{\infty} \leq \varepsilon
$$

there exists $y_{0} \in C^{2}(I, X)$ such that $D\left(y_{0}\right)=0$ and

$$
\left\|y-y_{0}\right\|_{\infty} \leq L \cdot \varepsilon
$$

A function $y$ satisfying (2) for some $\varepsilon>0$ is called an approximate solution of the equation $D(y)=0$. The number $L$ in the relation (3) is called an Ulam constant of $D$.

\section{Main Result}

The main result on Ulam stability for the operator $D$ defined by the relation (1) is contained in the next two theorems.

Theorem 1. Suppose that there exists $K>0$ such that

$$
g(x) \geq \frac{1}{K}, x \in I
$$

and the Riccati equation

$$
u^{\prime}=-u^{2}+f u+f^{\prime}-g
$$

has a solution $u \in C^{1}(I, \mathbb{R})$ with $u(a)=f(a)$. Then for every $y \in C^{2}(I, X)$ satisfying

$$
\|D(y)\|_{\infty} \leq \varepsilon,
$$

there exists $z \in C^{2}(I, X)$, such that $D(z)=0$ and

$$
\|y-z\|_{\infty} \leq K \varepsilon
$$

Proof. With the above $u$ satisfying (4), let

$$
U(x):=\int_{a}^{x} u(t) \mathrm{d} t, \varphi(x):=e^{U(x)}, x \in I,
$$

and

$$
F(x):=\int_{a}^{x} f(t) \mathrm{d} t, \quad \psi(x):=e^{F(x)-U(x)}, x \in I .
$$




\section{Consider the equation}

$$
y^{\prime \prime}(x)+f(x) y^{\prime}(x)+g(x) y(x)=h(x), \quad x \in I .
$$

Multiplying it by $\varphi(x)$, by using (4) and having in mind that $\varphi^{\prime}=u \varphi$, we get

$$
\left(\varphi y^{\prime}+(f-u) \varphi y\right)^{\prime}=\varphi h
$$

which leads to

$$
\varphi(x) \cdot y^{\prime}(x)+(f(x)-u(x)) \cdot \varphi(x) \cdot y(x)-y^{\prime}(a)=\int_{a}^{x} \varphi(t) h(t) \mathrm{d} t, x \in I .
$$

Multiplying the previous relation by $\frac{\psi(x)}{\varphi(x)}$, we obtain

$$
\psi(x) \cdot y^{\prime}(x)+\psi(x) \cdot(f(x)-u(x)) \cdot y(x)=\left(y^{\prime}(a)+\int_{a}^{x} \varphi(t) h(t) \mathrm{d} t\right) \frac{\psi(x)}{\varphi(x)}, x \in I .
$$

Since $\psi^{\prime}=(f-u) \psi$, it follows that

$$
(\psi y)^{\prime}(t)=\left(y^{\prime}(a)+\int_{a}^{t} \varphi(s) h(s) \mathrm{d} s\right) \cdot \frac{\psi(t)}{\varphi(t)}, t \in I,
$$

and this yields

$$
\psi(x) \cdot y(x)=y(a)+\int_{a}^{x}\left(y^{\prime}(a)+\int_{a}^{t} \varphi(s) h(s) \mathrm{d} s\right) \frac{\psi(t)}{\varphi(t)} \mathrm{d} t, x \in I .
$$

To resume, (7) is equivalent to

$$
y(x)=\frac{1}{\psi(x)}\left(y(a)+\int_{a}^{x}\left(y^{\prime}(a)+\int_{a}^{t} \varphi(s) h(s) \mathrm{d} s\right) \frac{\psi(t)}{\varphi(t)} \mathrm{d} t\right), x \in I
$$

In particular, the homogeneous equation

$$
z^{\prime \prime}(x)+f(x) z^{\prime}(x)+g(x) z(x)=0, x \in I
$$

is equivalent to

$$
z(x)=\frac{1}{\psi(x)}\left(z(a)+\int_{a}^{x} z^{\prime}(a) \frac{\psi(t)}{\varphi(t)} \mathrm{d} t\right), x \in I .
$$

Now let $\varepsilon>0$ and $y \in C^{2}(I)$ satisfying (5). Then $y$ satisfies also (7) and (8) with $|h(x)| \leq \varepsilon, x \in I$. Let $z \in C^{2}(I)$, be a solution of (9) with $z(a)=y(a)$ and $z^{\prime}(a)=y^{\prime}(a)$. According to (10),

$$
z(x)=\frac{1}{\psi(x)}\left(y(a)+\int_{a}^{x} y^{\prime}(a) \frac{\psi(t)}{\varphi(t)} \mathrm{d} t\right), x \in I
$$


and it satisfies the relation $D(z)=0$. So it remains to prove (6). Using (8) and (10), we obtain

$$
|y(x)-z(x)|=\frac{1}{\psi(x)}\left|\int_{a}^{x}\left(\int_{a}^{t} \varphi(s) h(s) \mathrm{d} s\right) \frac{\psi(t)}{\varphi(t)} \mathrm{d} t\right|, x \in I .
$$

Suppose that $x \in I, x>a$. Then

$$
|y(x)-z(x)| \leq \varepsilon \frac{1}{\psi(x)} \int_{a}^{x}\left(\int_{a}^{t} \varphi(s) \mathrm{d} s\right) \frac{\psi(t)}{\varphi(t)} \mathrm{d} t .
$$

If $x \in I, x<a$, then by (11) we obtain

$$
\begin{aligned}
|y(x)-z(x)| & =\frac{1}{\psi(x)}\left|\int_{x}^{a}\left(\int_{a}^{t} \varphi(s) h(s) \mathrm{d} s\right) \frac{\psi(t)}{\varphi(t)} \mathrm{d} t\right| \\
& \leq \frac{1}{\psi(x)} \int_{x}^{a}\left|\int_{t}^{a} \varphi(s) h(s) \mathrm{d} s\right| \frac{\psi(t)}{\varphi(t)} \mathrm{d} t \\
& \leq \varepsilon \frac{1}{\psi(x)} \int_{x}^{a}\left(\int_{t}^{a} \varphi(s) \mathrm{d} s\right) \cdot \frac{\psi(t)}{\varphi(t)} \mathrm{d} t \\
& =\varepsilon \frac{1}{\psi(x)} \int_{a}^{x}\left(\int_{a}^{t} \varphi(s) \mathrm{d} s\right) \cdot \frac{\psi(t)}{\varphi(t)} \mathrm{d} t .
\end{aligned}
$$

Therefore, we have

$$
|y(x)-z(x)| \leq \varepsilon \frac{1}{\psi(x)} \int_{a}^{x}\left(\int_{a}^{t} \varphi(s) \mathrm{d} s\right) \cdot \frac{\psi(t)}{\varphi(t)} \mathrm{d} t, x \in I .
$$

To finish the proof it remains to show that

$$
Q(x):=\int_{a}^{x}\left(\int_{a}^{t} \varphi(s) \mathrm{d} s\right) \frac{\psi(t)}{\varphi(t)} \mathrm{d} t-K \psi(x) \leq 0, x \in I .
$$

To this end, let us remark that $Q^{\prime}(x)=\frac{\psi(x)}{\varphi(x)} P(x)$, where

$$
P(x):=\int_{a}^{x} \varphi(s) \mathrm{d} s-K \varphi(x)(f(x)-u(x)), x \in I .
$$

Since $\varphi^{\prime}=\varphi \cdot u$ we have

$$
P^{\prime}(x)=\varphi(x)-K \cdot \varphi(x) \cdot u(x) \cdot(f(x)-u(x))-K \cdot \varphi(x) \cdot\left(f^{\prime}(x)-u^{\prime}(x)\right), x \in I,
$$

and using (4) we get

$$
P^{\prime}(x)=\varphi(x) \cdot(1-K g(x)) \leq 0, x \in I .
$$

Combined with $P(a)=0$, this yields $P(x) \geq 0$ for $x<a, x \in I$, and $P(x) \leq 0$, for $x>a, x \in I$, respectively. 
Accordingly, $Q^{\prime}(x) \geq 0$ for $x \in I, x<a$ and $Q^{\prime}(x) \leq 0$ for $x \in I, x>a$, respectively. Since $Q(a)=-K<0$, we conclude that $Q(x) \leq 0, x \in I$. Thus, (12) is proved and this finishes the proof of the theorem.

A similar result is presented in the following.

Theorem 2. Suppose that there exists $K>0$ such that

$$
g(x) \leq-\frac{1}{K}, x \in I
$$

and the equation

$$
u^{\prime}=-u^{2}+f u+f^{\prime}+g
$$

has a solution $u \in C^{1}(I, \mathbb{R})$, with $u(a)=f(a)$. Then the operator $D$ is Ulam stable with the Ulam constant $K$.

Proof. The proof is analogous to the proof of Theorem 1. Only (13) should be replaced by $P^{\prime}(x)=$ $\varphi(x) \cdot(1+K g(x)) \leq 0, x \in I$.

In what follows we obtain some direct consequences of the Theorems 1 and 2.

Corollary 1. Suppose that there exists $K>0$ such that

$$
g(x) \geq \frac{1}{K}, x \in I
$$

and the equation

$$
u^{\prime}=-u^{2}-g
$$

has a solution $u \in C^{1}(I, \mathbb{R})$, with $u(a)=0$ for some $a \in I$. Then the operator $D: C^{2}(I, X) \rightarrow C(I, X)$

$$
D(y)=y^{\prime \prime}+g y
$$

is Ulam stable with the Ulam constant $K$.

Corollary 2. Suppose that there exists $K>0$ such that

$$
g(x) \leq-\frac{1}{K}, x \in I
$$

and the equation

$$
u^{\prime}=-u^{2}+g
$$

has a solution $u \in C^{1}(I, \mathbb{R})$ with $u(a)=0$ for some $a \in I$. Then the operator $D: C^{2}(I, X) \rightarrow C(I, X)$

$$
D(y)=y^{\prime \prime}+g y
$$

is Ulam stable with the Ulam constant $K$.

The proof of the previous corollaries follows immediately by taking $f=0$ in the Theorems 1 and 2, respectively.

Remark 1. The particular case $g(x)=\lambda^{\prime}(x)-\lambda^{2}(x), \lambda \in C^{1}(I, \mathbb{R})$ being a periodic function, is studied in $[14,15]$ and corresponds to the Hill's equation.

Remark 2. The stability of the operator D defined by (1) depends on the existence of a global solution for the Riccati Equation (4). In the following is given an example where some appropriate conditions on the coefficients 
of the Equation (4) lead to the existence of such a solution. For this we will use the following result contained in [16].

Theorem 3. Let $I=[0,+\infty)$ and $a, b, c \in C(I, \mathbb{R}), x_{0} \in I$ and $y_{0} \geq 0$. If $a(x) \leq 0, c(x) \geq 0$ for all $x \in I$, then the Cauchy problem

$$
\left\{\begin{array}{c}
y^{\prime}=a(x) y^{2}+b(x) y+c(x), x \in I \\
y\left(x_{0}\right)=y_{0}
\end{array}\right.
$$

has a unique nonnegative solution $y=\varphi(x)$ on $\left[x_{0},+\infty\right)$.

Corollary 3. Let $I=[0,+\infty)$ and suppose that:

(i) $\quad f(0) \geq 0$;

(ii) $f^{\prime}(x) \geq g(x), x \in I$;

(iii) $g(x) \geq \frac{1}{K}>0, x \in I$.

Then the operator D defined by (1) is Ulam stable with the Ulam constant $K$.

Proof. The result is a simple consequence of the Theorems 1 and 3.

Analogously, we obtain the following result.

Corollary 4. Let $I=[0,+\infty)$ and suppose that:
i) $\quad f(0) \geq 0$;
ii) $f^{\prime}(x) \geq-g(x), x \in I$;
iii) $g(x) \leq-\frac{1}{K}<0, x \in I$.

Then the operator D defined by (1) is Ulam stable with the Ulam constant $K$.

Example 1. Let $I=[0,+\infty), 0<b<a$ and suppose that $b \leq|g(x)| \leq a, x \in I$. Then the operator $D$ defined by the relation

$$
D(y)(x)=y^{\prime \prime}(x)+a x y^{\prime}(x)+g(x) y(x), y \in C^{2}(I), x \in I,
$$

is Ulam stable with the Ulam constant $\frac{1}{b}$.

Proof. Let $f(x)=a x, x \in I$. The condition $b \leq|g(x)| \leq a, x \in I$ and the continuity of $g$ implies $g(x) \in[b, a], x \in I$, or $g(x) \in[-a,-b], x \in I$ (clearly, if $g$ has two values of opposite signs, then $g$ must take the value zero at some point in $I$ ).

If $g(x) \in[b, a], x \in I$, then, according to Corollary $3, D$ is Ulam stable with the Ulam constant $\frac{1}{b}$. If $g(x) \in[-a,-b], x \in I$, then $D$ is Ulam stable with the Ulam constant $\frac{1}{b}$, in view of Corollary 4.

\section{Conclusions}

In this paper, we studied the Ulam stability of a linear differential operator of second order acting on a Banach space. We proved the Ulam stability of the equation using the assumption that a certain Riccati differential equation has a global solution. In appropriate conditions of the coefficients of the operators, we obtained an explicit representation of its Ulam constant. Finally, we presented some stability results for particular cases of the operator.

Author Contributions: These authors contributed equally to this work. All authors read and agreed to the published version of the manuscript.

Funding: This work was partially supported by a grant of the Romanian Ministry of Research and Innovation, project number 10PFE/16.10.2018, Perform-Tech-UPT-The increasing of the institutional performance of the Polytechnic University of Timişoara by strengthening the research, development and technological transfer capacity in the field 
of "Energy, Environment and Climate Change, within Program 1-Development of the national system of Research and Development, Subprogram 1.2-Institutional Performance-Institutional Development Projects-Excellence Funding Projects in RDI, PNCDI III.

Acknowledgments: The authors would like to thank the referees for carefully reading of the manuscript and for giving useful comments and suggestions which improve the paper.

Conflicts of Interest: The authors declare no conflict of interest.

\section{References}

1. Hyers, D.H.; Isac, G.; Rassias, T. Stability of Functional Equations in Several Variables; Birkhäuser Boston Inc.: Basel, Switzerland, 1998.

2. Brzdȩk, J.; Popa, D.; Raşa, I.; Xu, B. Ulam Stability of Operators; Academic Press: Cambridge, MA, USA, 2018.

3. Obloza, M. Hyers stability of linear differential equation. Roczn. Nauk. Dydakt. Pr. Math. 1993, 13, $259-270$.

4. Alsina, C.; Ger, R. On some inequalities and stability results related to the exponential function. J. Inequal. Appl. 1998, 2, 373-380. [CrossRef]

5. Miura, T.; Miyajima, S.; Takahasi, S.E. Hyers-Ulam stability of linear differential operator with constant coeffcients. Math. Nachr. 2003, 258, 90-96. [CrossRef]

6. Popa, D.; Raşa, I. On the Hyers-Ulam stability of the linear differential equation. J. Math. Anal. Appl. 2011, 381, 530-537. [CrossRef]

7. Baias, A.B.; Popa, D. On the best Ulam constant of the second order linear differential operator. Rev. R. Acad. Cienc. Exactas Fís. Nat. Ser. A Mat. 2020, 114, 23. [CrossRef]

8. Onitsuka, M.; Shoji, T. Hyers-Ulam stability of first-order homogeneous linear differential equations with a real-valued coefficient. Appl. Math. Lett. 2017, 63, 102-108. [CrossRef]

9. Jung, S.-M. Hyers-Ulam stability of a system of first order linear differential equations with constant coefficients. J. Math. Anal. Appl. 2006, 320, 549-561. [CrossRef]

10. Miura, T.; Miyajima, S.; Takahasi, S.E. A characterization of Hyers-Ulam stability of first order linear differential operators. J. Math. Anal. Appl. 2003, 286, 136-146. [CrossRef]

11. Popa, D.; Raşa, I. Hyers-Ulam stability of the linear differential operator with nonconstant coefficients. Appl. Math. Comput. 2012, 219, 1562-1568. [CrossRef]

12. Takahasi, S.E.; Takagi, H.; Miura, T.; Miyajima, S. The Hyers-Ulam stability constants of first order linear differential operators. J. Math. Anal. Appl. 2004, 296, 403-409. [CrossRef]

13. Brzdęk, J.; Popa, D.; Raşa, I. Hyers-Ulam stability with respect to gauges. J. Math. Anal. Appl. 2017, 453, 620-628. [CrossRef]

14. Fukutaka, R.; Onitsuka M. Ulam Stability for a Class of Hill's Equations. Symmetry 2019, 11, 1483. [CrossRef]

15. Fukutaka, R.; Onitsuka, M. Best constant for Ulam stability of Hill's equations. Bull. Sci. Math. 2020, 163, 102888. [CrossRef]

16. Barbu V. Differential Equations, Springer: Berlin/Heidelberg, Germany, 2016.

(c) 2020 by the authors. Licensee MDPI, Basel, Switzerland. This article is an open access article distributed under the terms and conditions of the Creative Commons Attribution (CC BY) license (http:/ / creativecommons.org/licenses/by/4.0/). 